\title{
THE NON-LINEAR BREAK-UP OF AN INVISCID LIQUID JET USING THE SPATIAL-INSTABILITY METHOD
}

\author{
D. P. BUSKER \\ Volvo Car, Born, The Netherlands \\ and \\ A. P. G. G. LAMERS and J. K. NIEUWENHUIZEN \\ Department of Mechanical Engineering, Eindhoven University of Technology, Eindhoven, \\ The Netherlands
}

(Recelved 4 November 1987; accepted for publication 30 June 1988)

\begin{abstract}
A liquid jet originating from a nozzle with radius $r_{0}^{*}$ breaks up into dropletś in consequence of disturbances of certain frequencies, depending on the fluid properties and the nozzle geometry. A theoretical model is developed to describe the growth of these disturbances at the jet surface. The model is based on the inviscid and irrotational flow governed by the Laplace equation together with the kinematical and dynamical conditions at the free surface of the jet. A comparison is made between the model and experimental data from literature. The model predicts a dependence on the disturbance-amplitude of the break-off mode. Contrary to other experimental results, the model predicts satellites (i.e. smaller droplets between the main larger ones) at wavelengths exceeding a critical value of (10/7) $2 \Pi r_{0}^{*}$. The disturbances grow at wavelengths more than the theoretical bound of $2 \Pi r_{0}^{*}$. Discrepancies with experimental data are possible because of the neglect of the effect of viscosity in the theory. It is shown that the effect of viscosity on the jet can be neglected under cetain conditions.
\end{abstract}

\section{INTRODUCTION}

A liquid jet originating from a nozzle is sensitive to disturbances. Disturbances of certain frequencies cause the jet to break up into a series of successive droplets. There are two theoretical methods to investigate the behaviour of a disturbed liquid jet. The "spatial-instability" method describes the disturbance of the jet surface as a travelling wave in axial direction. The "temporal-instability" method describes the surface disturbance of the jet as a standing wave on an infinitely long cylinder with the nozzle at infinity.

The model presented in this paper is based on spatial instability and describes the jet form close to the nozzle in the form of travelling waves with harmonic influences. The Laplace equation together with the dynamical and kinematical boundary condition for the free surface are used to describe the radius of the jet and the velocity. The model is mathematically simplified by neglecting both the effects of viscosity and the surroundings. It is possible to approximate the solution of the equations for the radius of the jet by the solution of a simplified fluid dynamical theory, the Cosserat theory, which is a simplified one-dimensional theory. In this article we present an approximation for radius and velocity by Taylor series expansions with respect to the disturbance-amplitude.

The break-up process depends on the surface tension $\sigma^{*}$, density $\rho^{*}$, nozzle radius $r_{0}^{*}$ and initial disturbance-amplitude $\delta_{0}^{*}$. The disturbances grow with time and distance from the nozzle. Piezo-crystals or mechanical vibrators can be used as sources when applied to the jet surface, the velocity or the pressure distribution in the jet.
Previously published theoretical models are of a more limited use as the model presented in this paper, either because the stability analysis ignores higher harmonic effects or because these models are based on a simplified fluid dynamical theory. The spatial-instability method describes the physical reality better than the temporal-instability method does, whereas the first method does not impose periodic axial demands on the jet. The model based on spatial instability shows that satellites can break up before or after a main drop, dependent on the disturbanceamplitude. A condition is derived by which the influences of the viscosity can be estimated and eventually neglected.

\section{LITERATURE}

The first mathematical model was published by Rayleigh (1878). This model was a stability analysis for infinitely small disturbances of an inviscid jet based on the temporal-instability method. Weber (1931) and recently Sterling and Sleicher (1975) extended this analysis to an aerodynamically influenced viscous jet. According to these linearized models the only dependent variable for the break-up process is the break-up time.

By approximately 1960 these linearized theories were found inadequate to describe the phenomenon accurately. Only the break-up time could be reasonably well compared with experiments. At present the formation of satellites is considered to be more characteristic for the phenomenon than the break-up length or break-up time. Yuen (1968) and Lafrance (1974, 1975) have obtained analytical approximations for an 
inviscid jet ignoring influences of the environment on the basis of temporal instability and mass conservation for higher harmonic disturbances. They arrived at establishing the formation and the existence of satellites. Rutland and Jameson (1970) compared experimental data with numerical results based on Yuen's model. By coupling the initial and final volume of the droplets they succeeded in calculating diameters of representative satellites and main drops. They concluded that in case of wavelengths $\lambda^{*} \leqq(10 / 7) 2 \Pi r_{0}^{*}$ no satellites could be formed. With a modified model, Lafrance (1974) showed that no satellites were formed for $\lambda^{*} \leqq(10 / 8) 2 \Pi r_{0}^{*}$. These conclusions are certainly not correct in the case of viscous jets. Chaudhary and Maxworthy $(1980 a, b)$ and Chaudhary and Redekopp (1980) used a comparable model and performed experiments on the jet behaviour and satellite drop formation.

Keller et al. (1973) used spatial instability in combination with a stability analysis to describe the breakup process of a jet. As a result, the description of the break-up process is improved. From a mathematical viewpoint the disturbances behave like waves progressing on the jet surface. Bogy $(1978,1979 \mathrm{a}, \mathrm{b})$ expanded the Cosserat theory of Green (1976) to develop a new analytical model for higher harmonics based on spatial instability. The Cosserat theory is a one-dimensional theory simplifying the flow in the jet, especially in the radial direction.

The model presented in this paper is an extension of the models referred to above. It describes the break-up process of a jet with the spatial-instability method and mass conservation including higher harmonic effects. The jet form is approximated by a Taylor series expansion with respect to the disturbance-amplitude. The geometry of the jet, the velocity components in it, the break-up length and the break-up mode of satellites before or behind a main drop are calculated with our model. Computation of the last mentioned phenomenon is not found in earlier published articles. The model still has physical limitations on account of the neglect of viscosity and environment effects such as gravity, mass or heat transfer.

\section{MATHEMATICAL MODEL}

\section{General description}

A semi-infinite axisymmetric jet with liquid density $\rho^{*}$ and surface tension $\sigma^{*}$ emerges from a nozzle of radius $r_{0}^{*}$. The uniform velocity $v_{0}^{*}$ at the nozzle is harmonically disturbed. As a result the radius $R^{*}$ of the jet is a function of the axial coordinate $z^{*}$ and time $t^{*}$. Dimensional variables are indicated with the superscript *. Figure 1 shows the geometry of the jet. The characteristic length and time are $r_{0}^{*}$ and $t_{0}^{*}$ $=r_{0}^{*} / v_{0}^{*}$, respectively. The break-up process of the jet has a characteristic time-scale $t_{i}^{*}=\left(\rho^{*} r_{0}^{* 3} / \sigma^{*}\right)^{1 / 2}$. The characteristic dimensionless number is the Weber number which is defined as the quadratic ratio of the characteristic time scales $t_{1}^{*}$ and $t_{0}^{*}$ :

$$
W e=\left(t_{1}^{*} / t_{0}^{*}\right)^{2}=\rho r_{0}^{*}\left(v_{0}^{*}\right)^{2} / \sigma^{*} \text {. }
$$

The influence of the surroundings, viscosity and gravity are neglected. Rotational symmetry and irrotational flow in the jet are assumed. The flow in the jet is described by means of the Laplace equation, a pressure continuity condition at the jet boundary and a containment boundary condition, in the sense that all liquid remains within the jet surface. The nozzle diameter is constant and the axial jet velocity at the nozzle is sinusoidally disturbed.

The local jet radius $R^{*}$ and the velocity potential $\Phi^{*}$ are approximated by series expansions. The nonlinear differential equations are reduced to a set of linear differential equations; these are solved up to the third order to enable the description of satellites. From the homogeneous differential equations the characteristic dispersion relation is derived. The dispersion relation is solved numerically on a mainframe Burroughs. The break-up length $z_{B}^{*}$ and the local jet radius $R^{*}\left(z^{*}, t^{*}\right)$ are calculated by the computer. These results arc comparcd with the experimental data taken from the literature (Chaudhary and Maxworthy, 1980a, b).

\section{Formulation}

A velocity potential $\Phi^{*}$ with $u^{*}=\frac{\partial \Phi^{*}}{\partial z^{*}}$ and $v^{*}=\frac{\partial \Phi^{*}}{\partial r^{*}}$ is introduced. All variables are made dimensionless with respect to the characteristic time $t_{0}^{*}=r_{0}^{*} / v_{0}^{*}$ and the characteristic length $r_{0}^{*}$. The Laplace equation in terms of dimensionless $\Phi$ is

$\nabla^{2} \Phi=\Phi_{r r}+\frac{1}{r} \Phi_{r}+\Phi_{z z}=0 \quad\left(0 \leqq r \leqq R, 0 \leqslant z \leqslant z_{B}\right)$.

The kinematical boundary condition for free surface of

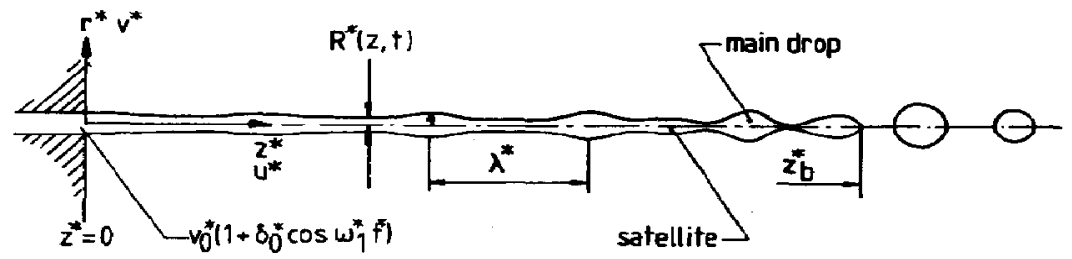

Fig. 1. Sketch of the geometry of a liquid jet emerging from a nozzle at $z^{*}=0$. The entrance velocity is disturbed sinusoidally. The local jet radius $R^{*}$ is a function of the axial coordinate and time. Under certain conditions the jet breaks up into main drops, spaced at wavelength $\lambda^{*}$, and satellites at the break-up length $z_{B}^{*}$. 
the jet is

$$
\Phi_{r}=R_{t}+\Phi_{z} R_{z} \quad(r=R) .
$$

The dynamical boundary condition is given by Busker (1983):

$$
\begin{aligned}
\Phi_{1}+\frac{1}{2}\left(\Phi_{r}^{2}+\right. & \left.\Phi_{z}^{2}\right)+\frac{1}{W e}\left\{1 /\left[R\left(1+R_{z}^{2}\right)^{1 / 2}\right]\right. \\
& \left.-R_{z z} /\left(1+R_{z}^{2}\right)^{3 / 2}\right\}=\frac{1}{2}+\frac{1}{W e} \quad(r=R) .
\end{aligned}
$$

The nozzle diameter is constant:

$$
R=1 \quad(z=0)
$$

and the jet velocity is disturbed sinusoidally according to

$$
\Phi_{z}=1+\delta_{0} \cos \omega_{1} t \quad(z=0,0 \leqq r \leqq R) .
$$

Chaudhary and Redekopp (1980) started from the same eqs [(2)-(4)]. Our approach, however, is to seek to find solutions of the form

$$
R=1+R^{\prime}=1+\delta_{\mathrm{o}} \eta_{1}+\delta_{\mathrm{o}}^{2} \eta_{2}+\delta_{\mathrm{o}}^{3} \eta_{3}+O\left(\delta_{\mathrm{o}}^{4}\right)
$$

and

$$
\Phi(r, z, t)=z+\delta_{0} \Phi_{1}+\delta_{0}^{2} \Phi_{2}+\delta_{0}^{3} \Phi_{3}+O\left(\delta_{0}^{4}\right)
$$

Here $\eta_{i}$ is the ith-order harmonic surface distortion, and $\Phi_{i}$ is the $i$ th-order harmonic velocity potential of the main disturbance with $i=1$. The derivative of the velocity potential on the boundary is transferred to the undisturbed jet geometry $R=1$ by a re-expansion of the velocity potential $\Phi$ into a series of the boundary transformation $R^{\prime}$. After substitution of the boundary transformation $R^{\prime}$ the tcrms of cqs (2)-(6) can be expressed in terms of $\delta_{0}^{0}, \delta_{0}^{1}, \delta_{0}^{2}$ and $\delta_{0}^{3}$, respectively. After that, the equations are separated into powers of $\delta_{0}$. The zeroth-order solution gives the undisturbed cylindrical jet.

The first-order equations are given by

$$
\begin{gathered}
\nabla^{2} \Phi_{1}=0 \quad(0 \leqq r \leqq 1) \\
\Phi_{1, r}-\eta_{1, r}-\eta_{1, z}=0 \quad(r=1) \\
-\Phi_{1, t}-\Phi_{1, z}+\frac{1}{W e}\left(\eta_{1}+\eta_{1, z z}\right)-0 \quad(r=1)
\end{gathered}
$$

with the following nozzle conditions:

$$
\begin{aligned}
\eta_{1}=0 & (z=0) \\
\Phi_{1, z}=\cos \omega_{1} t & (z=0,0 \leqq r \leqq 1) .
\end{aligned}
$$

From these first-order equations a stability rule, the dispersion relation, will be derived in the next paragraph. The second-order $\left(\delta_{0}^{2}\right)$ and the third-order $\left(\delta_{0}^{3}\right)$ equations are given by Busker (1983).

\section{Dispersion relation}

Because of the first-order nozzle conditions [see eqs (12) and (13)] and eq. (9) which is defined at $r=0$, a first-order solution is assumed which has the form

$$
\begin{aligned}
\eta_{1}=C e^{i(\omega t-k z)} & (z, t \geqq 0) \\
\Phi_{1}=D I_{0}(k r) e^{i(\omega t-k z)} & (z, t \geqq 0, r \leqq 1) .
\end{aligned}
$$

Here, $I_{n}(x)$ is the modified Bessel function of the first kind and $n$th order. $\Phi_{1}$ is a solution of the Laplace equation.

Substitution of $\eta_{1}$ and $\Phi_{1}$ into eqs (10) and (11) produces the following characteristic equation:

$$
(\omega-k)^{2}=\frac{k\left(k^{2}-1\right)}{W e} \frac{I_{1}(k)}{I_{0}(k)} .
$$

This equation is called the dispersion relation, because it connects the frequency $\omega$ to the wave-number $k$ at a given Weber number. The real part of the wavenumber is related to the wavelength $\lambda$ by

$$
\operatorname{Re}(k)=\frac{2 \Pi}{\lambda}
$$

The imaginary part of $k$ determines the rate of growth of the disturbance. The dispersion relation has an infinite number of solutions for $k$ per $\omega$. The solutions for $k$ are functions of $\omega$ and $W e$. Keller et al. (1973) gives the zeroth-order solutions for this equation:

$$
(\omega-k)^{2}=O\left(W e^{-1}\right)
$$

leading to $k=\omega+O\left(W e^{-1 / 2}\right)$, and

$$
\frac{I_{\mathrm{o}}(k)}{I_{1}(k)}=O\left(\mathrm{We}^{1}\right)
$$

leading to $k={ }^{ \pm} i j_{v n}+O\left(W e^{-1}\right)$ with $I_{0}\left({ }^{ \pm} i, j_{v n}\right)=0$.

Keller et al. (1973) gives an approximation for the solution of eqs (18) and (19), respectively:

$$
\begin{aligned}
k_{1,2}=\omega \pm & {\left[\frac{\omega\left(\omega^{2}-1\right)}{W e} \frac{I_{1}(\omega)}{I_{o}(\omega)}\right]^{1 / 2}+O\left(W e^{-1}\right) } \\
k_{3,4 n}={ }^{ \pm} i j_{o n} & +\frac{j_{o n}\left(j_{o n} j_{o n}+1\right)}{W e\left(\omega^{2}+j_{o n}^{2}\right)^{2}}\left[2 \omega j_{o n} \mp i\left(\omega^{2}-j_{o n}^{2}\right)\right] \\
+ & O\left(W e^{-2}\right) .
\end{aligned}
$$

Another solution is (private communication of J. Boersma, Department of Mathematics, Eindhoven University of Technology)

$$
k_{5}=W e-\left(2 \omega-\frac{1}{2}\right)+\frac{\left(9 / 8+\omega-3 \omega^{2}\right)}{W e}+O\left(W e^{-2}\right) .
$$

Only those solutions of $k$ which have a positive phasevelocity $c=\omega / \operatorname{Re}(k)>0$ and a positive group-velocity $c_{g}=\frac{\partial \omega}{\partial[\operatorname{Re}(k)]}>0$ are valid solutions because only then energy is transported downstream and the waves are travelling in the same direction. The wave-numbers $k_{1,2}$ come up to these conditions. Here it is assumed that the disturbance excitator is at the nozzle and therefore energy goes downstream in the same manner as the waves. Other assumptions are possible depending on the place of the disturbance source along the jet but they are beyond the scope of this article. A singularity-point exists for $\omega=\omega_{s}$, because there is an abrupt change over from complex to real wave-number values. The dispersion relation has now two equal solutions. For $\omega<\omega_{\text {s }}$ the two solutions are 
conjugate complex (Fig. 2). By approximation we find for the singularity-point $\omega_{s}$ that

$$
\omega_{s} \approx 1-\frac{I_{1}(1)}{2 W e I_{0}(1)} \approx 1-\frac{0.221}{W e}
$$

Substitution of eq. (23) in eq. (20) gives for $k_{1,2}$ the expression

$$
k_{1,2} \approx 1+\frac{I_{1}(1)}{2 W e I_{0}(1)} \approx 1+\frac{0.221}{W e}
$$

Moreover the following relations hold:

$$
\begin{aligned}
\lim _{\omega \rightarrow \omega_{s}} \operatorname{Im}\left(k_{1,2}\right)=0 ; & \lim _{\omega \uparrow \omega_{s}} \frac{\partial \operatorname{Im}\left(k_{1,2}\right)}{\partial \omega}= \pm \infty ; \\
& \lim _{\omega \downarrow \omega_{s}} \frac{\partial \operatorname{Im}\left(k_{1,2}\right)}{\partial \omega}=0 .
\end{aligned}
$$

The maximum growth rate occurs at $\omega_{\text {opt }} \approx$ $0.4858^{1 / 2} \approx 0.6969$ for large Weber numbers.

\section{Effect of viscosity}

The dispersion relation for a jet with viscosity $\mu$ under the same conditions as an inviscid jet is given by Busker (1983):

$$
\begin{aligned}
(\omega-k)^{2}= & +\frac{k\left(k^{2}-1\right)}{W e} \frac{I_{1}(k)}{I_{0}(k)}-\frac{2 i(\omega-k) k}{R e}\left[2 k-\frac{I_{1}(k)}{I_{0}(k)}\right] \\
& -\frac{4 k^{3}}{R e^{2}}\left[k-\frac{l I_{0}(l) I_{1}(k)}{I_{1}(l) I_{0}(k)}\right]
\end{aligned}
$$

with $l^{2}=k^{2}+i(\omega-k) R e$.

In the case of $\omega<1$ and by approximating $\frac{I_{1}(k)}{I_{0}(k)} \approx \frac{k}{2}$ $+O\left(k^{2}\right)$ eq. (26) can be rewritten analogously to Weber (1931) as

$$
(\omega-k)^{2}=+\frac{k\left(k^{2}-1\right)}{W e} \frac{I_{1}(k)}{I_{0}(k)}-\frac{3 i(\omega-k) k^{2}}{R e} .
$$

The solution for this equation is

$$
\begin{aligned}
k_{1,2}= & \omega \pm\left[\omega\left(\omega^{2}-1\right) \frac{I_{1}(\omega)}{I_{0}(\omega)}\right]^{1 / 2}\left[\frac{1}{W e^{1 / 2}}\right. \\
& \left.+\frac{\left(3 i \omega^{2}+2\right)}{\left(3 i \omega^{2}-1\right) R e}\right] .
\end{aligned}
$$

The influence of the viscosity might be neglected if the influence of the viscous terms are less than $2 \%$ of the surface tension term, thus

$$
\left|\frac{\left(3 i \omega^{2}+2\right) W e^{1 / 2}}{\left(3 i \omega^{2}-1\right) R e}\right|<0.02
$$

For the examples of the experiment of Chaudhary and Maxworthy $(1980 a, b)$ this gives in the case of $\omega_{1}$ $=0.4313, W e=922.6$ and $R e=1486$ a value about $3.7 \%$ and in case of $\omega_{1}=0.720, W e=330.9$ and $R e=889.9$ a value about $2.8 \%$ (see Application).

\section{First-order solution}

The dispersion relation provides the wave-number values $k_{1,2}$ for a given dimensionless frequency $\omega_{1}$ and Weber number which are used in the first-order solutions (14) and (15):

$$
\begin{aligned}
& \eta_{1}=P_{1} \cos \theta_{1}+P_{2} \cos \theta_{2} \\
& \Phi_{1}=Q_{1} I_{0}\left(k_{1} r\right) \sin \theta_{1}+Q_{2} I_{0}\left(k_{2} r\right) \sin \theta_{2}
\end{aligned}
$$

where $\theta_{1}=\omega_{1} t-k_{1} z, \theta_{2}=\omega_{1} t-k_{2} z$, and $P_{1}, P_{2}, Q_{1}$ and $Q_{2}$ are constant. Substitution into the kinematical boundary condition and the nozzle conditions produces the following first-order solution:

$$
\begin{gathered}
\eta_{1}=\frac{P}{V}\left(\cos \theta_{1}-\cos \theta_{2}\right) \\
\Phi_{1}=\frac{P}{V}\left[\frac{-\left(\omega_{1}-k_{1}\right) I_{0}\left(k_{1} r\right)}{k_{1} I_{1}\left(k_{1}\right)} \sin \theta_{1}\right. \\
\left.+\frac{\left(\omega_{1}-k_{2}\right) I_{0}\left(k_{2} r\right)}{k_{2} I_{1}\left(k_{2}\right)} \sin \theta_{2}\right]
\end{gathered}
$$

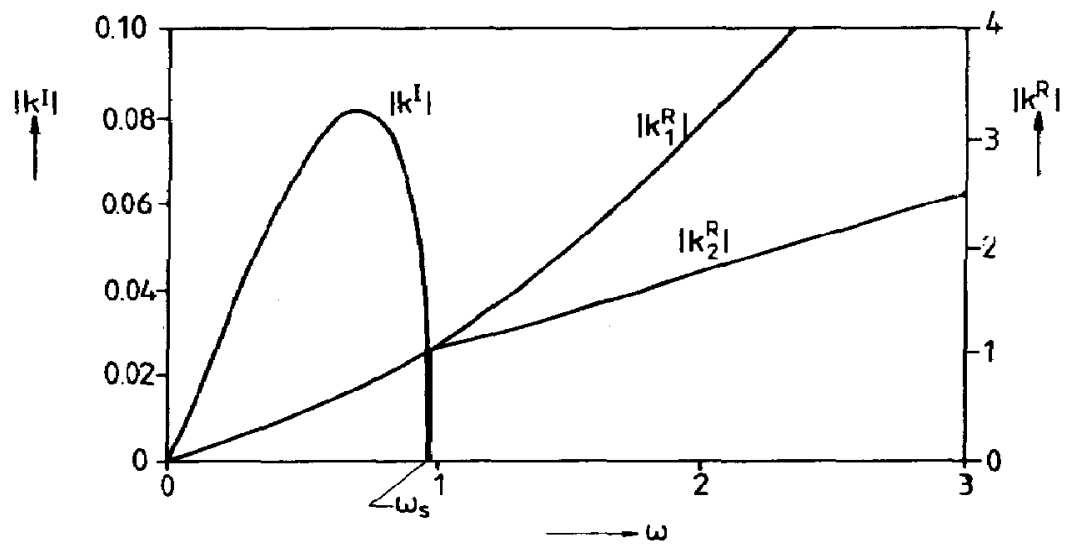

Fig. 2. Solutions of the characteristic dispersion relation at $W e=10$. The path of wave-number $k$ as a function of the frequency $\omega$ is given. A singularity-point exists at $\omega=\omega_{s}$. In case of $\omega<\omega_{s}$ the solutions of $k$ are complex conjugated. 
Here $P=I_{1}\left(k_{1}\right) I_{1}\left(k_{2}\right)$ and $V=\left(\omega_{1}-k_{1}\right) I_{1}\left(k_{2}\right)-\left(\omega_{1}\right.$ $\left.-k_{2}\right) I_{1}\left(k_{1}\right)$.

Second-and third-order solutions

The second-and third-order solutions are derived from the second- and third-order differential equations together with the nozzle conditions. They consist of a particular part and a homogeneous part. The particular parts of the second-order boundary differential equations are composed of terms with $\eta_{1}$ and $\Phi_{1}$. In the case of the third-order equations it is composed of terms with $\eta_{1}, \Phi_{1}, \eta_{2}$ and $\boldsymbol{\Phi}_{2}$. By substituting the lower-order solutions in the particular part of the second- and third-order differential equations a general form of the particular second- and third-order solution is derived. These general forms for the second- and third-order solutions are substituted in the homogeneous parts of the boundary differential equations. The particular solutions are then determined. As homogeneous solutions of the ithorder ( $i=2$ or 3 ) are taken:

$$
\begin{aligned}
\eta_{i}^{H} & =C_{1} \cos \theta_{i 1}+C_{2} \cos \theta_{i 2} \\
\Phi_{i}^{H} & =D_{1} I_{0}\left(k_{1 i}\right) \sin \theta_{i 1}+D_{2} I_{0}\left(k_{2 i}\right) \sin \theta_{i 2}
\end{aligned}
$$

with $\theta_{2 i}=2 \omega_{1} t-k_{2 i} z$ and $\theta_{3 i}=3 \omega_{1} t-k_{3 i} z$.

$k_{2 i}$ and $k_{3 i}$ come from the dispersion relation with $\omega=2 \omega_{1}$ and $3 \omega_{1}$, respectively. The homogeneous solutions are substituted in the boundary differential equations. A relationship between $\eta_{i}^{H}$ and $\Phi_{i}^{H}$ is deduced. The complete solution is found by substitution of the homogeneous and particular parts of the solution in the nozzle conditions. The equations with which all constants necessary for the complete solution can be calculated are given by Busker (1983). In the case of $\omega_{1}<\omega_{s}$ and $k_{1,2}$ values are conjugate complex and it is possible to make simplifications in $R$ and $\Phi$. The third-order solution is given in Appendix 1 .

If necessary the velocity components, pressure distribution and volume in the jet could be calculated up to the break-up point. The field of interest in this paper is restricted to the unstable growth modes of the disturbances, i.e. $\omega_{1}<\omega_{s}$. Because $R(z)$ is continuous, $R$ cannot describe the discontinuous behaviour of the jet beyond the break-up point. Therefore only $z \leqq z_{B}$ is considered.

\section{APPLICATION}

Comparing the model with a particular experiment

Chaudhary and Maxworthy $(1980 \mathrm{a}, \mathrm{b})$ investigated experimentally the break-up process with a piezoelement as a disturbance source. This was driven by a sinusoidal wave at a frequency $f^{*}=100 \mathrm{kHz}$ with a peak voltage $V_{e}^{*}$ as amplitude. The jet velocity $v_{0}^{*}$ was fitted to reach the desired wavelength $\lambda^{*}$. It resulted in the dimensionless wave-number

$$
k=\frac{2 \pi r_{0}^{*}}{\lambda^{*}}
$$

Chaudhary and Redekopp (1980) used a temporalinstability model to determine the dimensionless minimum break-up time $T_{B}$. In this article a relation is deduced between the theoretical disturbance-amplitude $\delta_{0}$ and the peak voltage $V_{e}^{*}$ of their experiments. The results for the experimental break-off mode are compared with the predictions of the present model. The experimental data are transformed to desirable values of $\omega_{1}, W e$ and $z_{B}$ for the spatial-instability model with the relationships

$$
\begin{gathered}
v_{0}^{*} \approx \lambda^{*} f^{*} \\
W e \approx \frac{2 \pi^{2} \rho^{*} r_{0}^{*}\left(f^{*}\right)^{2}}{\sigma^{*}\left(k^{*}\right)^{2}}
\end{gathered}
$$

and

$$
z_{B}=\left(W e T_{B}\right)^{1 / 2}
$$

The theoretical value of $\omega_{1}$ is found by a variation of $\omega$ in the dispersion relationship at the given Weber number to the effect that $\operatorname{Re}\left(k_{1,2}\right)=k$.

The Reynolds number in the jet is given by

$$
R e=\frac{\rho^{*} v_{o}^{*} r_{0}^{*}}{\mu^{*}} \approx \frac{2 \pi \rho^{*}\left(r_{0}^{*}\right)^{2} f^{*}}{\mu^{*} k} .
$$

Chaudhary and Maxworthy (1980a) used a water jet and varied $k$ between 0.3 and 1 . The values of the physical quantities are given in Table 1 . This resulted in values of $W e$ in the range of $170-1910$ and values of $R e$ in the range of 640-2140. A series of experimental data have been compared with a series of numerical results of our model. The wave-number $k=0.4312$ is used which gives $W e=922.6, R e=1486$ and $\omega_{1}=0.4313$. Some pictures are given by Chaudhary (1980a). In the case of $W e=922.6$ the experimental amplitude $V_{e}^{*}$ varied between 1 and $80 \mathrm{~V}$ and the corresponding minimum break-up length $z_{B}$ between 860 and 305. A break-up length smaller than 586 causes a satellite break-up after the main drop, as opposed to the break-up before the main drop for higher values of $z_{B}$. The corresponding calculated values of $z_{B}$ lead to a range of dimensionless disturbance-amplitude values $\delta_{0}$ between $3 \times 10^{-5}$ and 4 $\times 10^{-2}$ (Table 2).

$V_{e}^{*}$ and $\delta_{0}$ can be written in a lugarithmic linear relation. The constants are calculated by linear regres-

Table 1. Values of the physical quantities used with the experiments of Chaudhary and Maxworthy (1980a, b)

\begin{tabular}{lcl}
\hline $\begin{array}{l}\text { Physical } \\
\text { quantity }\end{array}$ & Value & Dimension \\
\hline$r_{0}^{*}$ & $3.048 \times 10^{-5}$ & $\mathrm{~m}$ \\
$\sigma^{*}$ & $65.3 \times 10^{-3}$ & $\mathrm{~N} / \mathrm{m}^{3}$ \\
$\rho^{*}$ & 1002 & $\mathrm{~kg} / \mathrm{m}^{3}$ \\
$\mu^{*}$ & $9.128 \times 10^{-4}$ & $\mathrm{~Pa} \mathrm{~s}$ \\
\hline
\end{tabular}


Table 2. Experimental data of Chaudhary and Maxworthy for the peak voltage $V_{e}^{*}$ and the break-up length $z_{B}$ compared with the calculated values of $z_{B}$ and the disturbance-amplitude $\delta_{0}$ at the dimensionless frequency $\omega_{1}=0.4313$ and Weber number $W e=922.6$

\begin{tabular}{|c|c|c|c|}
\hline \multicolumn{2}{|c|}{$\begin{array}{c}\text { Experiments of Chaudhary } \\
\text { and Maxworthy }\end{array}$} & \multicolumn{2}{|c|}{$\begin{array}{c}\text { Calculated results of } \\
\text { the model }\end{array}$} \\
\hline$V_{e}^{*}$ & $z_{B}$ & $z_{B}$ & $\delta_{\mathrm{o}}$ \\
\hline 80 & 305 & 298 & $5 \times 10^{-3}$ \\
\hline 70 & 321 & 324 & $4 \times 10^{-3}$ \\
\hline 40 & 397 & 399 & $2 \times 10^{-3}$ \\
\hline 30 & 437 & 434 & $15 \times 10^{-4}$ \\
\hline 20 & 497 & 493 & $9 \times 10^{-4}$ \\
\hline 15 & 533 & 537 & $6 \times 10^{-4}$ \\
\hline 10 & 586 & 584 & $4 \times 10^{-4}$ \\
\hline 4 & 703 & 694 & $15 \times 10^{-5}$ \\
\hline 2 & 784 & 779 & $7 \times 10^{-5}$ \\
\hline 1 & 860 & 876 & $3 \times 10^{-5}$ \\
\hline
\end{tabular}

sion leading to

$$
\ln \left(V_{e}^{*}\right)=9.26+0.896 \ln \left(\delta_{0}\right)
$$

A second series of experimental data with $k=0.720$ and $W e=330.9$ is used as a comparison. The experimental values of $z_{B}$ were between 417 and 169 . The calculated values of $\delta_{0}$ with $\omega_{1}=0.720$ are in the range between $3 \times 10^{-5}$ and $3 \times 10^{-3}$ (Table 3 ).

The same $\log$-linear relation for $V_{e}^{*}$ and $\delta_{0}$ does not apply when break-up lengths are smaller than 300 (Fig. 3).

Break-up mode of main drops and satellites

Another test criterion for the validity of the model is the break-off mode of main drops and satellites at the minimum break-up length $z_{B \min }$. For small amplitudes
Table 3. Experimental data of Chaudhary and Maxworthy for the peak voltage $V_{e}^{*}$ and the break-up length $z_{B}$ compared with calculated values of $z_{B}$ and the disturbance-amplitude $\delta_{0}$ at the dimensionless frequency $\omega_{1}=0.720$ and Weber number $W e=330.9$

\begin{tabular}{cccc}
\hline $\begin{array}{c}\text { Experiments of Chaudhary } \\
\text { and Maxworthy }\end{array}$ & \multicolumn{2}{c}{$\begin{array}{c}\text { Calculated results of } \\
\text { the model }\end{array}$} \\
\hline$V^{*}$ & $z_{B}$ & $z_{B}$ & $\delta_{0}$ \\
\hline 70 & 175 & 173 & $3 \times 10^{-3}$ \\
50 & 196 & 193 & $2 \times 10^{-3}$ \\
30 & 228 & 231 & $1 \times 10^{-3}$ \\
20 & 256 & 257 & $6 \times 10^{-4}$ \\
15 & 270 & 268 & $5 \times 10^{-4}$ \\
10 & 291 & 295 & $3 \times 10^{-4}$ \\
7 & 311 & 314 & $2 \times 10^{-4}$ \\
2 & 379 & 379 & $6 \times 10^{-5}$ \\
1 & 417 & 417 & $3 \times 10^{-5}$ \\
\hline
\end{tabular}

$\delta_{0}$ the main drop will break off first of all at the breakup length $z_{B}=z_{B \min }$ followed by the satellite at $z_{B}>z_{B \min }$. For large amplitudes $\delta_{0}$ the satellite will break off first of all at $z_{B}=z_{B \min }$ followed by the main drop. The jet shape in the surroundings of the breakup length $z_{B}$ for small and large $\delta_{0}$ values are shown in Figs 4 and 5 .

In the case of $\omega_{1}=0.4313$ and $W e=922.6$ the experimental transition value of the different modes occurs for $V_{e}^{*}$ between 4 and $10 \mathrm{~V}$ (Chaudhary and Maxworthy, 1980a) corresponding with $\delta_{0}$ between $15 \times 10^{-6}$ and $4 \times 10^{-4}$ for the fixed $\log$-linear relation between $V_{e}^{*}$ and $\delta_{0}$. The analytical model predicts that the transition value for $\delta_{0}$ lies between 5 $\times 10^{-3}$ and $6 \times 10^{-3}$. This discrepancy is probably due to the neglect of viscous effects in the jet (Re

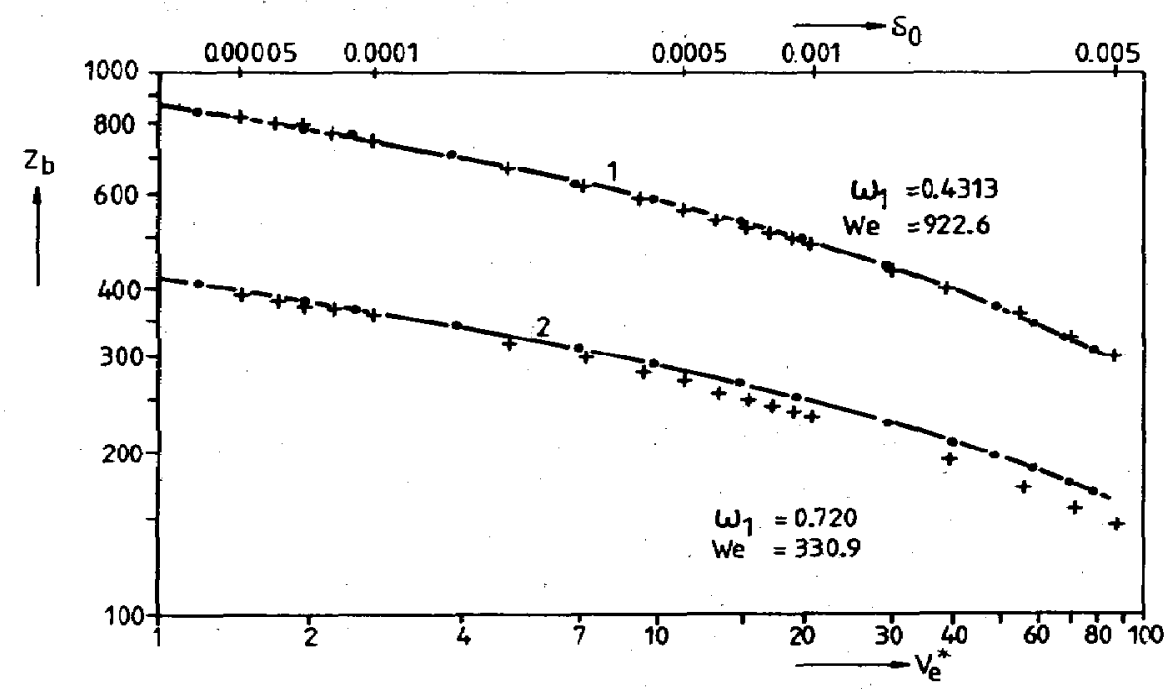

Fig. 3. Comparison between calculated values of the break-up length $z_{H}(+)$ with experimental data from Chaudhary and Maxworthy $(1980 \mathrm{a}, \mathrm{b})(0)$. Curve 1 represents values of $z_{B}$ in case of $W e=992.6$ and $\omega_{1}$ $=0.4313$. Curve 2 in case of $W e=330.9$ and $\omega_{1}=0.720$ under the conditions of eq. (41). 


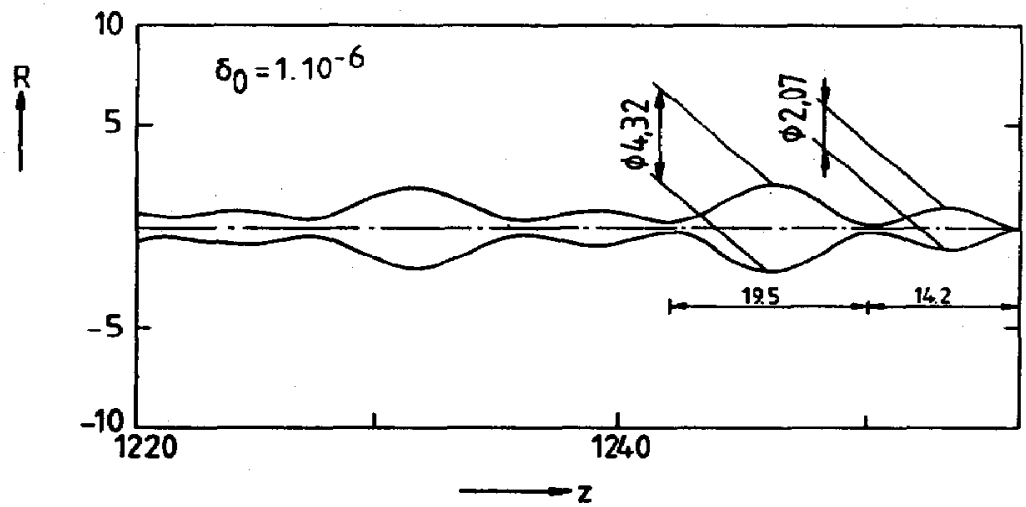

Fig. 4. Calculated jet shape just before the break-up point $z_{B}$ for small values of the disturbance-amplitude $\delta_{0}$. The calculated diameters and their lengths of the main drop and satellite are given.

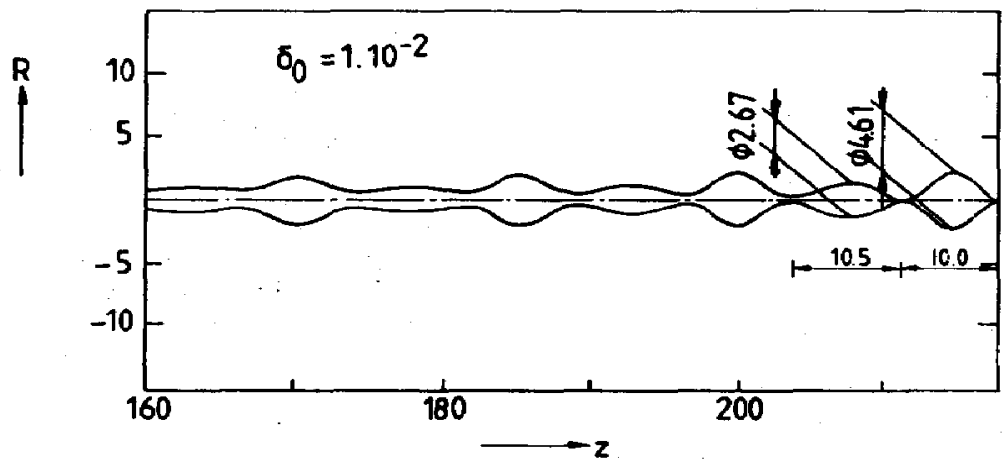

Fig. 5. Calculated jet shape just before the break-up point $z_{B}$ for large values of the disturbance-amplitude $\delta_{0}$. The calculated diameters and the lengths of the main drop and satellite are given.

=1486). The reason for this is the slow specific velocity of the break-up process $\left(\sigma^{*} / \rho^{*} r_{0}^{*}\right)^{1 / 2}$, in which the viscosity effects cannot be neglected. The model of Chaudhary and Maxworthy (1980a) seems to predict the break-off mode.

\section{The forming of satellites}

Sterling and Sleicher (1975) demonstrated the unstable break-up for viscous liquid jets with aerodynamic effects. Their model and the model described in this article do not predict the forming of satellites for $\omega_{1} \geqq 0.7$. Lafrance (1974) and Rutland and Jameson (1970) did not succeed in finding satellites either. Figure 6 shows the divergence between the model and experiments.

Consistent with the model of Yuen (1968) our model predicts the formation of two satellites for $\omega_{1} \leqq 0.3$ as a result of the conjugate complex $k$ values for the second- and third-order solutions of the dispersion relation (Fig. 7). For long wavelengths more than one satellite will be formed. When the analytical model is extended to higher orders (larger than three) more satellites are formed at suitable values of $\omega_{1}$.
Effect of the disturbance-amplitude on the dimensions of satellites and the main drop

The volume of satellites and main drops is dependent on the disturbance-amplitude $\delta_{0}$. The ratio of the maximum diameters of satellites and main drops changes the same way as the ratio of their lengths changes with $\delta_{0}$. These phenomena can be seen in Figs 4 and 5. The ratio of the diameters of the satellite to the main drop for $\delta_{0}=1 \times 10^{-2}$ is 0.579 while the ratio of the lengths is 1.05 . In case of $\delta_{0}=1 \times 10^{-6}$ the ratio of the diameters becomes 0.479 and the ratio of the lengths 0.728 . This means that satellites are larger for greater values of $\delta_{0}$. About this no experimental data are available. Because of the limited use of this model, this point will not be pursued further.

\section{Break-up length as function of the frequency}

The dependence of the minimum break-up length $z_{B}$ on the frequency for several Weber and amplitudenumbers is shown in Fig. 8. The plot shows that the minimum break-up length over the whole range $0<\omega_{1}<\omega_{s}$ occurs somewhere between $\omega_{1}=0.7$ and $\omega_{1}=0.8$ for small $\delta_{0}$, and moves towards $\omega_{s}$ for large 


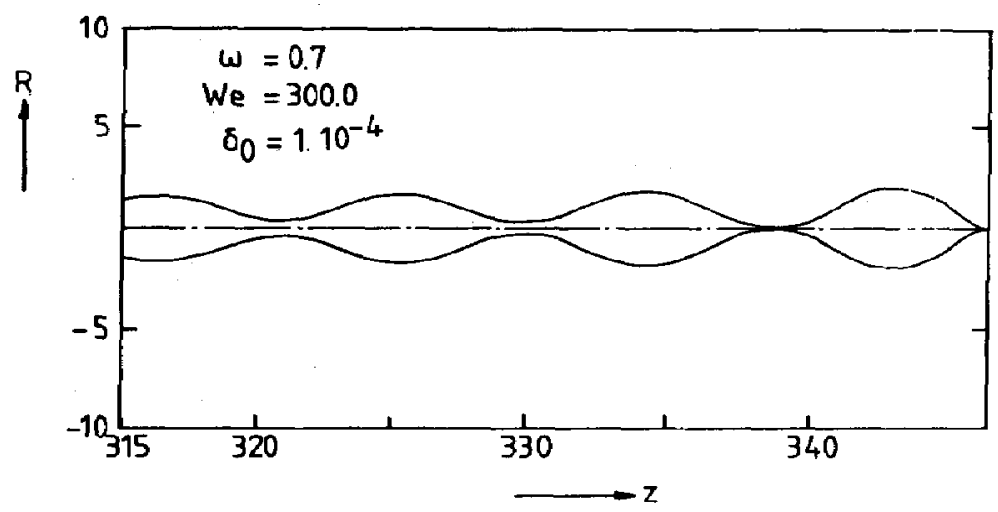

Fig. 6. Calculated jet shape at $W e=300, \omega=0.7$ and $\delta_{o}=1 \times 10^{-4}$ shows no forming of satellites.

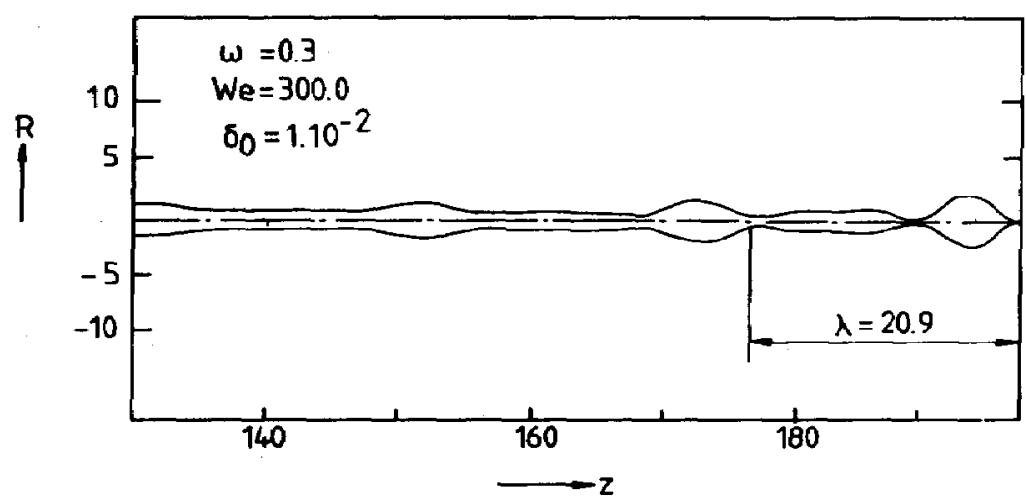

Fig. 7. Forming of two satellites under the conditions of $W e=300$ and $\delta_{0}=1 \times 10^{-2}$ in the case of the dimensionless frequency $\omega_{1}=0.3$. The calculated wavelength is given.

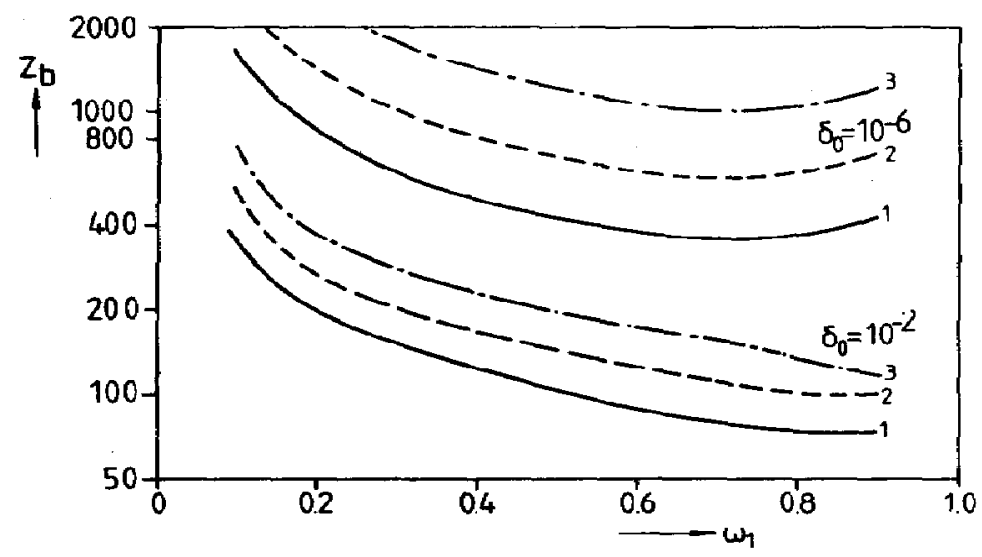

Fig. 8. Values of the break-up length $z_{B}$ as a function of the dimensionless frequency $\omega_{1}$ with the disturbance-amplitude $\delta_{o}$ and $W e$ as parameters. (1) Lines with $W e=100$, (2) lines with $W e=300$, and (3) lines with $\mathrm{We}=1000$.

$\delta_{0}$. It can be expected that an undisturbed jet picking up a disturbance from its surroundings will not break up at the most unstable mode (just below $\omega_{1}=0.7$ ) but that it breaks up at frequencies in an interval $0.65<\omega_{1}<0.8$.

\section{DISCUSSION AND CONCLUSIONS}

The theoretical model of spatial instability provides a good insight into the break-up process in a jet and may be extended. In some respects the model is in accordance with the results of experiments published 
in literature, in other respects it shows discrepancies therewith. The model describes the physical reality better than models based on temporal instability. Our model and Yuen's (1968) are analogous and have as common features the forming of two satellites for $\omega_{1}<\frac{1}{3}$ and the absence of satellites for $\omega_{1}>\frac{7}{10}$.

The model is more extensive than the one-dimensional Cosserat theory because we made use of twodimensional fluid mechanical equations taking account of the flow in radial direction. However, by comparing the theoretical predictions with available experimental data, it follows that the discrepancies exist due to the neglect of the viscosity or higher-order terms of the solution. Further research could take into account the effect of the nozzle shape and the disturbances on the break-up mode of the jet. In addition, the effects of gravity, aerodynamical, mass and heat transfer phenomena could be investigated.

\section{NOTATION}

c dimensionless phase velocity

$c_{g} \quad$ dimensionless group velocity

C constant of eq. (14)

$C_{1}, C_{2}$ constants of eq. (34)

$D$ constant of eq. (15)

$D_{1}, D_{2}$ constants of eq. (35)

$f^{*} \quad$ frequency, $1 / \mathrm{s}$

$I_{n}(x) \quad$ modified Bessel function of the first kind and the $n$ th-order

Im (. . .) imaginary part of an indicated variable

$j_{o n} \quad$ roots of $I_{0}\left(i, j_{o n}\right)=0$

$k$ dimensionless wave-number in eqs (14)-(16), (26) and (27)

$k_{1,2}$ dimensionless wave-numbers in eqs (20), (24) and (28)

$k_{3,4 n}$ dimensionless wave-numbers in eq. (21)

$k_{5}$ dimensionless wave-number in eq. (22)

$O($. .) order of terms of the indicated variable

$P \quad$ numerator of eqs (32) and (33)

$P_{i, j} \quad$ constants of eq. (42); subscript $i$ indicates the $i$ th order and subscript $j$ the number of the constant

$P_{1}, P_{2}$ constants of eq. (30)

$Q_{1}, Q_{2}$ constants of eq. (31)

$r$

$r *$

$r_{\mathbf{0}}^{*}$

$\boldsymbol{R}$

$R^{*}$

$\boldsymbol{R}^{\prime}$

dimensionless radial distance

radial distance, $m$

nozzle radius, characteristic length, m

dimensionless local jet radius

local jet radius, $m$

boundary transformation

$\operatorname{Re} \quad \operatorname{Reynolds}$ number $\left(R e=\frac{\rho^{*} v_{\mathrm{o}}^{*} r_{0}^{*}}{\mu^{*}}\right)$

$\operatorname{Re}(.$.$) real part of the indicated variable$

$t^{*} \quad$ time, $\mathrm{s}$

$t_{0}^{*} \quad$ characteristic time, $s$

$t_{1}^{*} \quad$ characteristic time-scale, $\mathrm{s}$

$T_{B} \quad$ dimensionless minimum break-up time

$u^{*}$

$v^{*}$

$v_{0}^{*}$
$V$

$V \stackrel{*}{*}$

We

$z$

$z^{*}$

$z_{B}^{*}$

$Z_{\text {Bmin }}$

denominator in eqs (32) and (33)

experimental voltage, $V$

Weber number $\left(W e=\frac{\rho^{*} r_{0}^{*}\left(v_{0}^{*}\right)^{2}}{\sigma^{*}}\right)$

dimensionless axial distance

axial distance, $m$

break-up length, $m$

dimensionless minimum break-up length

Greek letters

$\delta_{\mathrm{o}} \quad$ dimensionless disturbance-amplitude

$\eta_{i} \quad$ ith-order harmonic surface distortion

$\theta_{1}, \theta_{2}$ constants of eqs (30), (31), (32), (33) and (42)

$\theta_{2 i}, \theta_{3 i}$ constants of eqs (34), (35) and (42)

$\lambda^{*} \quad$ wavelength, $\mathrm{m}$

$\mu^{*} \quad$ viscosity, Pa s

$\rho^{*} \quad$ density, $\mathrm{kg} / \mathrm{m}^{3}$

$\sigma^{*} \quad$ surface tension, $\mathrm{N} / \mathrm{m}$

$\Phi$ dimensionless velocity potential

$\Phi_{i} \quad$ ith-order harmonic velocity potential

$\omega, \omega_{1}$ dimensionless frequency

$\omega_{\text {opt }}$ dimensionless frequency with maximum growth rate

$\omega_{s} \quad$ singular value of the dimensionless frequency

\section{Subscripts}

$B$ break-up value

$r$ derivative with respect to the radial variable $r$

$t$ derivative with respect to the time $t$

$z$ derivative with respect to the axial variable $z$

\section{Superscripts}

$H$ homogeneous part of the solution

I imaginary part

$R \quad$ real part

* dimensional quantity

\section{REFERENCES}

Bogy, D. B., 1978, Use of one-dimensional Cosserat-theory to study instability in a viscous jet. Phys. Fluids 21, 190-197.

Bogy, D. B., 1979a, Break-up of a liquid jet; second perturbation solution for one-dimensional Cosserat-theory, IBM J. Res. Dev. 23, 87-92.

Bogy, D. B., 1979b, Break-up of a liquid jet: third perturbation solution, Phys. Fluids 22, 224-230.

Busker, D. P., 1983, An analytical model for a liquid jet which breaks up into droplets. Master's Thesis, Eindhoven University of Technology, Eindhoven (in Dutch).

Chaudhary, K. C. and Maxworthy, T., 1980a, The non-linear capillary instability of a liquid jet. Part 2, experiments on the jet behaviour before droplet formation. J. Fluid Mech. 96, 275-286.

Chaudhary, K. C. and Maxworthy, T., 1980b, The non-linear capillary instability of a liquid jet. Part 3 , experiments on satellite drop formation and control. J. Fluid Mech. 96, 287-297.

Chaudhary, K. C. and Redekopp, L. C., 1980, The non-linear capillary instability of a liquid jet. Part 1 , theory. $J$. Fluid Mech. 96, 257-274.

Green, A. E., 1976, On the non-linear behaviour of fluid jets. Int. J. Engng Sci. 14, 49-63.

Keller, J. B., Rubinow, S. F. and Tu, Y. O., 1973, Spatial instability of a jet. Phys. Fluids 16, 2052-2055. 
Lafrance, $P$., 1974, Non linear break-up of a liquid jet. Phys. Fluids 17, 1913-1914.

Lafrance, P., 1975, Non-linear break-up of a laminar jet. Phys. Fluids 18, 428-432.

Rayleigh, 1878, On the stability of jets. Proc. Lond. math. Soc. 11, 4-15.

Rutland, D. F. and Jameson, G. J., 1970, Theoretical prediction of the size of drops formed in the break-up of capillary jets. Chem. Engng Sci. 25, 1689-1698.

Sterling, A. M. and Sleicher, C. A., 1975, The instability of capillary jets. J. Fluid Mech. 68, 477-495.

Weber, C. 1931, Zum Zerfall eines Flussigkeitsstrahles. $Z$. angew. Math. Mech. 4, 136-154.

Yuen, M. C., 1968, Non-linear capillary instability of a liquid jet. J. Fluid Mech. 3, 151-163.

\section{APPENDIX 1}

The third-order solution for the jet surface is

$$
\begin{aligned}
R=1 & +2 \delta_{0}\left[P_{1,1} \operatorname{Im}\left(\cos \theta_{1}\right)\right] \\
& +\delta_{0}^{2} \operatorname{Re}\left(\left\{P_{2,1} \cos \theta_{21}+P_{2,2} \cos \theta_{22}+2 P_{2,3} \cos 2 \theta_{1}\right.\right.
\end{aligned}
$$

$$
\begin{aligned}
& \left.\left.+P_{2,3} \cos \left[2\left(\omega_{1} t-k^{R} z\right)\right]+P_{2,6}\left[\cosh \left(2 k^{I} z\right)-1\right]\right\}\right) \\
& +\delta_{0}^{3} \operatorname{Im}\left(\left\{\sum_{i=1}^{2} P_{3, i} \cos \theta_{3 i}+2 \sum_{i=1}^{2}\left[P_{3,4 i} \cos \left(\theta_{2 i}+\theta_{1}\right)\right.\right.\right. \\
& \left.+P_{3,(4 i+1)} \cos \left(\theta_{2 i}-\theta_{1}\right)\right]+2 P_{3,12} \cos \left(3 \theta_{1}\right) \\
& +2 P_{3,14} \cos \left(2 \theta_{1}+\theta_{2}\right)+2 P_{3,15} \cos \left(2 \theta_{1}-\theta_{2}\right) \\
& \left.\left.+\left(2 P_{3,18}+P_{3,20}\right) \cos \theta_{1}+P_{3,21} \cos \theta_{2}\right\}\right)
\end{aligned}
$$

with $\theta_{1}=\omega_{1} t-k_{1} z$ and $\theta_{2}=\omega_{1} t-k_{2} z$.

The subscripts $i$ and $j$ of the constants $P_{i, j}$ indicate the $i$ th order and the number of the constant, respectively.

To obtain numerical results for the analytical solution of the jet-surface it is necessary to calculate the $k$ values for the first-, second- and third-order equations using $\omega_{1}$ and the Weber number as parameters. The calculation of the various constants $P_{r, j}$ is the next step. After that the jet surface $R$ can be determined for different amplitudes $\delta_{0}$ and time $t$ in a given distance interval $\Delta z$. From these results computer-plots can be made. A description of the software is given by Busker (1983). 